\title{
Instability of the negative mass Schwarzschild naked singularity
}

\author{
Reinaldo J. Gleiser and Gustavo Dotti \\ Facultad de Matemática, Astronomía y Física, Universidad Nacional de Córdoba, \\ Ciudad Universitaria, (5000) Córdoba, Argentina*
}

\begin{abstract}
We study the negative mass Schwarzschild spacetime, which has a naked singularity, and show that it is perturbatively unstable. This is achieved by first introducing a modification of the well known Regge - Wheeler - Zerilli approach to black hole perturbations to allow for the presence of a "kinematic" singularity that arises for negative masses, and then exhibiting exact exponentially growing solutions to the linearized Einstein's equations. The perturbations are smooth everywhere and behave nicely around the singularity and at infinity. In particular, the first order variation of the scalar invariants can be made everywhere arbitrarily small as compared to the zeroth order terms. Our approach is also compared to a recent analysis that leads to a different conclusion regarding the stability of the negative mass Schwarzschild spacetime. We also comment on the relevance of our results to the stability of more general negative mass, nakedly singular spacetimes.
\end{abstract}

PACS numbers: 04.50.+h,04.20.-q,04.70.-s, 04.30.-w

\section{INTRODUCTION: THE NEGATIVE MASS SCHWARZSCHILD SOLUTION}

The positive mass theorems of general relativity establish that, under plausible assumptions, asymptotically flat solutions of the Einstein equations with physically acceptable matter sources cannot have negative total mass. Another well known feature of general relativity, compatible with the positivity theorems, is the possibility of the presence, or eventual formation, of singularities in the spacetime manifold. However, in the most relevant examples where this happens, the singularity is surrounded by a horizon, so that it is unobservable to an observer outside the horizon. That this should happen generically in all physically relevant situations is the essential content of the versions of the Cosmic Censorship hypothesis. On the other hand, from a strictly mathematical point of view, there is plenty of solutions of the Einstein equations that are asymptotically flat and present a negative total mass, but, in general, they are also nakedly singular, which would make them physically unacceptable. A simple and particularly interesting example of this type of solutions is the negative mass Schwarzschild spacetime. Nevertheless, for different reasons, these solutions are not expected to arise from physically acceptable regular initial data. One should notice, however, that what is physically acceptable may change as more data is available. What we have in mind here is the recent suggestion of the existence of "phantom matter", that is some field that carries negative kinetic energy, as a possible explanation for the observed acceleration of the scale factor of the Universe [1].

A different, but equally important consideration regarding negative masses is the manner

*Electronic address: gdotti@famaf.unc.edu.ar 
in which they interact with positive masses. This gives rise to different types of instabilities that have been analyzed in the literature. We refer to [2] for a recent review. It is rather remarkable, however, that until recently [2], the stability of the negative mass Schwarzschild solution, that, in a loose sense, may be considered as representing the field of an isolated negative mass source, had not been considered in the literature, although the essential machinery for this analysis, at least at the linear level, had been available for some time. The analysis carried out in [2] leads to the conclusion that if certain boundary conditions, assumed suitable for the problem, are imposed, the negative mass Schwarzschild singularity should be considered as stable under linear perturbations. However, as discussed in Section VII, the marginally stable solution of Zerilli's equation used to prove stability in [2], is only a twice differentiable functions of the radial coordinate. As a consequence, the associated perturbed metric is not continuous and thus it is not a solution of the linearized vacuum Einstein's equations (it actually satisfies the field equations of a singular distribution of sources). Given this situation, we undertake in this paper a new analysis of the linear stability of the negative mass Schwarzschild solution. This is based on a modification of the standard Regge-Wheeler-Zerilli formalism (reviewed in Section II) that simplifies and makes more transparent the analysis of the perturbative equations for negative mass. This is given in Section III. Next, we consider the existence of "zero modes", i.e., static solutions of the perturbation equations. We give the general solution, that for each mode depends on two integration constants, and prove that there is no choice of these constants such that the solution can be considered as a "small" perturbation. We give also a physical interpretation for these solutions that supports our conclusions. In Section VI, based on this modified form of the formalism, and this is the main result presented in this paper, we prove that under quite general boundary conditions, all scalar perturbative modes are unstable. Our results and possible consequences are finally summarized in Section VIII.

\section{PERTURBATION EQUATIONS FOR THE SPHERICALLY SYMMETRIC SCHWARZSCHILD SOLUTION.}

The vacuum solution of Einstein's equations for the exterior of a bounded spherically symmetric source, first obtained by Schwarzschild, may be written in the form,

$$
d s^{2}=-\left(1-\frac{2 M}{r}\right) d t^{2}+\left(1-\frac{2 M}{r}\right)^{-1} d r^{2}+r^{2}\left(d \theta^{2}+\sin ^{2}(\theta) d \phi^{2}\right)
$$

where $\theta$ and $\phi$ are standard coordinates on the unit sphere, and $M$ is a real (positive or negative) constant, that can be identified with the Newtonian mass of the source. The range of $r$ is limited by the singularities of the metric coefficients in (1). For $M>0$ the singularity at $r=2 M$ corresponds to a regular horizon, and the solution may be extended to $r<2 M$. The resulting space time is known as a "black hole". The horizon hides a curvature singularity for $r=0$, rendering the portion of space time outside the horizon globally hyperbolic. Similarly, for negative mass $(M<0)$ there is a singularity for $r=0$, but in this case there is no horizon, the singularity is "naked", and the resulting space time is not globally hyperbolic.

The rather strange properties of the spacetime described by (1) led to question the possibility of their physical realization. Since the solution (at least for $r>2 M$ ) is static, a first step in this direction, carried out by Regge and Wheeler [3], and completed later by 
Zerilli [4], was aimed at establishing their stability, through an analysis of the behavior of linear perturbations. The Einstein equations lead in this case to a set of coupled linear partial differential equations for the functions representing the perturbations. Because of the spherical symmetry of (1) this functions may be grouped into "even" (or scalar) and "odd" (or vector) sets that do not couple to each other, and are characterized by their dependence on the angular coordinates $(\theta, \phi)$. Still, the resulting perturbative equations contain, in general, some redundant information that can be eliminated through a choice of "gauge". A particular choice, that keeps full generality, was selected in [3], [4] and is known as the "Regge-Wheeler gauge". Independently of the gauge issue, the linear stability of the Schwarzschild space time, well known for $M>0$, was also established for vector perturbations for $M<0$ in [2]. For this reason we shall restrict our analysis to the scalar perturbations. In the Regge-Wheeler gauge, the scalar (even) perturbations, for each angular mode $(\ell, m)$, are fully described by four functions $H_{0}(r, t), H_{1}(r, t), H_{2}(r, t)$ and $K(r, t)$, in terms of which the perturbed metric takes the form,

$$
\begin{aligned}
d s^{2}= & -\left(1-\frac{2 M}{r}\right)\left(1-\epsilon H_{0} Y_{\ell, m}\right) d t^{2}+2 \epsilon H_{1} Y_{\ell, m} d t d r+\left(1-\frac{2 M}{r}\right)^{-1}\left(1+\epsilon H_{2} Y_{\ell, m}\right) d r^{2} \\
& +r^{2}\left(1+\epsilon K Y_{\ell, m}\right)\left(d \theta^{2}+\sin ^{2}(\theta) d \phi^{2}\right)
\end{aligned}
$$

where $\epsilon$ is an auxiliary parameter, useful in keeping track of the order of the perturbation. $Y_{\ell, m}=Y_{\ell, m}(\theta, \phi)$ are standard spherical harmonics on the sphere, and, on account of the spherical symmetry, we may restrict to $m=0$ without loss of generality. The linearized Einstein equations for the metric (2) imply $H_{0}(r, t)=H_{2}(r, t)$, and a set of coupled differential equations for $H_{1}, H_{2}$ and $K$. This can be solved in terms of the Zerilli function $\psi_{Z}(r, t)$, by the replacements,

$$
\begin{aligned}
K & =f(r) \psi_{Z}+\left(1-\frac{2 M}{r}\right) \frac{\partial \psi_{Z}}{\partial r} \\
H_{1} & =h(r) \frac{\partial \psi_{Z}}{\partial t}+r \frac{\partial^{2} \psi_{Z}}{\partial t \partial r} \\
H_{2} & =\frac{\partial}{\partial r}\left[\left(1-\frac{2 M}{r}\right)\left(h(r) \psi_{Z}+r \frac{\partial \psi_{Z}}{\partial r}\right)\right]-K
\end{aligned}
$$

where,

$$
\begin{aligned}
f(r) & =\frac{\lambda(\lambda+1) r^{2}+3 \lambda M r+6 M^{2}}{r^{2}(\lambda r+3 M)} \\
h(r) & =\frac{\lambda r^{2}-3 \lambda r M-3 M^{2}}{(r-2 M)(\lambda r+3 M)} \\
\lambda & =\frac{(\ell-1)(\ell+2)}{2}
\end{aligned}
$$

provided $\psi_{Z}$ is a solution of the Zerilli equation,

$$
\frac{\partial^{2} \psi_{Z}}{\partial t^{2}}-\frac{\partial^{2} \psi_{Z}}{\partial r_{*}^{2}}+V(r) \psi_{z}=0
$$

where,

$$
V(r)=2\left(1-\frac{2 M}{r}\right) \frac{\lambda^{2} r^{2}[(\lambda+1) r+3 M]+9 M^{2}(\lambda r+M)}{r^{3}(\lambda r+3 M)^{2}},
$$


and $r_{*}$ is the "tortoise" coordinate, related to $r$ by,

$$
\frac{d r_{*}}{d r}=\left(1-\frac{2 M}{r}\right)^{-1}
$$

In what follows, for simplicity, we choose the integration constant relating $r$ and $r_{*}$ such that,

$$
r_{*}=r+2 M[\ln (r-2 M)-\ln (2|M|)]
$$

Therefore, for $M<0$, we have $r_{*}=0$ for $r=0$.

We notice that the relations (3) can be inverted, giving,

$$
\psi_{Z}(r, t)=\frac{r(r-2 M)}{(\lambda+1)(\lambda r+3 M)}\left(H_{2}-r \frac{\partial K}{\partial r}\right)+\frac{r}{\lambda+1} K
$$

Therefore, there is a one to one correspondence between $\psi_{Z}$ and the Regge-Wheeler gauge functions, $H_{0}, H_{1}, H_{2}$ and $K$.

For the stability analysis one considers a time dependence of the form,

$$
\psi_{Z}(t, r)=e^{i \omega t} \psi(r)
$$

which turns (5) into the Schrödinger-like form,

$$
-\frac{d^{2} \psi_{Z}}{d r_{*}^{2}}+V(r) \psi=\omega^{2} \psi
$$

Very loosely speaking, stability is attained if (11) has no solutions with negative $\omega^{2}$. More rigorously, this statement must be supplemented with the imposition of appropriate boundary conditions on $\psi(r)$. The resulting boundary value problem for $M>0$ turns out to be quite different from that for $M<0$.

The case $M>0$ has been considered in detail in the literature (see, e.g. [5]). In this case we have $-\infty<r_{*}<+\infty$ and $V(r)$ is positive definite in this range. Then, imposing boundary conditions that imply that the perturbed space time remains asymptotically flat, with a regular horizon, it can be shown that (11)is turned into a self adjoint boundary value problem, that admits only solutions with $\omega^{2}>0$, and, therefore, the spacetime is linearly stable.

For $M<0$ the problem is quite different. The domain in this case is $0 \leq r_{*}<+\infty$ and, besides the singularity of $V$ in (6)at $r=r_{*}=0$, there is another one at the interior point $r=-3 M / \lambda$. Also, it is not at all clear what boundary conditions are physically adequate for $r_{*}=0$. Finally, Zerilli's auxiliary function $\psi_{Z}(t, r)$ is generically singular (at $r=-3 M / \lambda)$ for smooth metric perturbations. An attempt at an appropriate formulation of the problem was recently given in [2]. Unfortunately, for reasons explained below in Section VII, we consider that there are some difficulties in that analysis that require a revision of their conclusions.

\section{A MODIFIED REGGE-WHEELER-ZERILLI FORMALISM}

In view of the difficulties indicated in the previous Section, we present here a slight modification of the Regge-Wheeler-Zerilli formalism that, as we shall show, allows for a 
simple analysis of the stability problem for $M<0$, with the intriguing result that these solutions are unstable under arbitrarily small perturbations. Following [2], we define $\mu=$ $-2 M$, but consider, instead of $\psi$, the function,

$$
\zeta(t, r)=(2 \lambda r-3 \mu) \psi_{Z}(t, r) .
$$

From (5) we easily find that $\zeta$ satisfies the equation,

$$
\frac{\partial^{2} \zeta}{\partial t^{2}}=\mathcal{O} \zeta
$$

where

$$
\begin{aligned}
\mathcal{O} \zeta \equiv \frac{(r+\mu)^{2}}{r^{2}}\left(\frac{\partial^{2} \zeta}{\partial r^{2}}\right)-\frac{\left(4 \lambda r^{2}+6 \lambda \mu r-3 \mu^{2}\right)(r+\mu)}{r^{3}(2 \lambda r-3 \mu)} & \frac{\partial \zeta}{\partial r} \\
& -\frac{(r+\mu)\left(3 \mu^{2}-6 \lambda \mu r+4 \lambda^{2} r^{2}\right)}{r^{4}(2 \lambda r-3 \mu)} \zeta
\end{aligned}
$$

Note that $\zeta(t, r)$ is also simply related to $H_{1}, H_{2}$, and $K$ :

$$
\begin{aligned}
K= & \frac{(\mu+r) r\left(\frac{\partial \zeta}{\partial r}\right)+(\lambda r-\mu) \zeta}{r^{2}(2 \lambda r-3 \mu)} \\
H_{1}= & \frac{2 r(\mu+r)\left(\frac{\partial^{2} \zeta}{\partial t \partial r}\right)+\mu\left(\frac{\partial \zeta}{\partial t}\right)}{r(r+\mu)(2 \lambda r-3 \mu)} \\
H_{2}= & \frac{(r+\mu)}{(2 \lambda r-3 \mu)}\left(\frac{\partial^{2} \zeta}{\partial r^{2}}\right)-\frac{4 \lambda r^{2}+6 \lambda \mu r-3 \mu^{2}}{2 r(2 \lambda r-3 \mu)^{2}}\left(\frac{\partial \zeta}{\partial r}\right) \\
& +\frac{3 \mu^{2}-6 \lambda \mu r+4 \lambda^{2} r^{2}}{2 r^{2}(2 \lambda r-3 \mu)^{2}} \zeta(t, r)
\end{aligned}
$$

and shares with $\psi_{Z}(t, r)$ its uniqueness and gauge invariance properties. We may regard (14) as a map $\Psi:=\zeta \rightarrow\left(K, H_{1}, H_{2}\right)$, a possible inverse map being

$$
\zeta(t, r)=-\left(\frac{2 r^{2}(r+\mu)}{\lambda+1}\right) \frac{\partial K}{\partial r}+\left(\frac{(2 \lambda r-3 \mu) r}{\lambda+1}\right) K+\left(\frac{2 r(r+\mu)}{\lambda+1}\right) H_{2}
$$

The following results follow straightforwardly from the definition of $\Psi$, eqn (14):

Lemma 1: the metric perturbation given by $\Psi(\zeta(t, r))$ is $C^{\infty}$ (for $r \in(0, \infty)$ ) if and only if $\zeta(t, r)$ is $C^{\infty}$ and satisfies the condition

$$
\left.\frac{\partial \zeta(t, r)}{\partial r}\right|_{r=3 \mu /(2 \lambda)}=\left.\frac{2 \lambda^{2}}{3 \mu(2 \lambda+3)} \zeta(t, r)\right|_{r=3 \mu /(2 \lambda)}
$$

If (13) is solved for initial data $\zeta\left(t=t_{o}, r\right)$ and $\partial \zeta / \partial t\left(t=t_{o}, r\right)$ both satisfying (16), the solution will satisfy (16) for all $t$ where it is defined. In other words, (16) is preserved by the evolution equation (13)

Proof: if the metric perturbation $\left(K, H_{1}, H_{2}\right)$ is $C^{\infty}$ then so is $\zeta$, as follows from (15), and we can use Taylor's theorem in (14) to prove (16) is required to assure that $\left(K, H_{1}, H_{2}\right)$ are 
smooth at $r=3 \mu /(2 \lambda)$. The preservation of this constraint by the evolution equation (13) follows from the fact that the operator $\mathcal{O}$ defined in (13) sends functions satisfying (16) to functions satisfying (16)

Since the coefficients in (13) depend only on $r$, we may separate variables by setting $\zeta(t, r)=\exp (i \omega t) \chi(r)$, which gives

$$
\begin{aligned}
\frac{d^{2} \chi}{d r^{2}}= & \frac{\left(4 \lambda r^{2}+6 \lambda r \mu-3 \mu^{2}\right)}{(2 \lambda r-3 \mu)(r+\mu) r} \frac{d \chi}{d r} \\
& +\left[\frac{\left(4 \lambda^{2} r^{2}-6 \lambda r \mu+3 \mu^{2}\right)}{(2 \lambda r-3 \mu)(r+\mu) r^{2}}-\frac{r^{2}}{(r+\mu)^{2}} \omega^{2}\right] \chi
\end{aligned}
$$

This ODE, obviously, does not have the Schrödinger like form of (11). Moreover, the coefficients of $\chi$ and $d \chi / d r$ are singular at $r=3 \mu /(2 \lambda)$. However, the nature of this singularity is rather subtle, and, as shown below, all solutions of (17) are regular around $r=3 \mu /(2 \lambda)$, irrespective of the boundary conditions for $r=0$ and $r=\infty$.

Lemma 2: For any $\lambda, \mu$ and $\omega$, all solutions of (13) are regular at $r=3 \mu /(2 \lambda)$.

Proof: Let $\rho=r-3 \mu /(2 \lambda)$, then expanding (17) around $\rho=0$ we get

$$
\begin{array}{r}
0=\frac{d^{2} \chi}{d \rho^{2}}+\left(-2 \rho^{-1}-\frac{4 \lambda^{2}}{3 \mu(3+2 \lambda)}+\frac{16(3+\lambda) \lambda^{3} \rho}{9 \mu^{2}(3+2 \lambda)^{2}}+\mathcal{O}\left(\rho^{2}\right)\right) \frac{d \chi}{d \rho} \\
+\left(\frac{-4 \lambda^{2}}{3 \mu(3+2 \lambda) \rho}-\frac{16 \lambda^{4}-81 w^{2} \mu^{2}}{9 \mu^{2}(3+2 \lambda)^{2}}+\frac{8 \lambda^{2}\left(4 \lambda^{3}+27 w^{2} \mu^{2}\right) \rho}{9 \mu^{3}(3+2 \lambda)^{3}}+\mathcal{O}\left(\rho^{2}\right)\right) \chi(\rho) \\
\equiv \frac{d^{2} \chi}{d \rho^{2}}+\left(\rho^{-1} \sum_{k \geq 1} a_{k} \rho^{k-1}\right) \frac{d \chi}{d \rho}+\left(\rho^{-1} \sum_{k \geq 1} b_{k} \rho^{k-1}\right) \chi(\rho)
\end{array}
$$

If $\chi$ is analytic around $\rho=0$ :

$$
\chi(\rho)=\sum_{k \geq 0} c_{k} \rho^{k}
$$

then (18) reduces to

$$
s\left(s-1+a_{1}\right) c_{s}+\sum_{l=0}^{s-1} c_{l}\left(l a_{s+1-l}+b_{s-l}\right)=0, \quad s=1,2,3, \ldots
$$

In our case $a_{1}=-2$ so $(20)$ splits into a system of three equations in the unknowns $c_{o}, c_{1}$ and $c_{2}$ and an infinite system on the $c_{k}, k>2$. Since the determinant of the finite system is

$$
\left|\begin{array}{ccc}
b_{1} & a_{1} & 0 \\
b_{2} & b_{1}+a_{2} & 2 a_{1}+2 \\
b_{3} & a_{3}+b_{2} & b_{1}+2 a_{2}
\end{array}\right|=0
$$

(for all $\mu, \lambda$ and $\omega$ ), and the infinite system can be solved iteratively in terms of $c_{3},(20)$ has a solution for arbitrary values of $c_{o}$ and $c_{3}$, i.e., two linearly independent solutions (for more details on this special case of singular point see e.g. [9], pp.205-215) 


\section{STATIC SOLUTIONS}

Static solutions correspond to $\omega=0$. In this case $(17)$ is reduced to,

$$
\frac{d^{2} \chi}{d r^{2}}-\frac{\left(4 \lambda r^{2}+6 \lambda r \mu-3 \mu^{2}\right)}{(2 \lambda r-3 \mu)(r+\mu) r} \frac{d \chi}{d r}-\frac{\left(4 \lambda^{2} r^{2}-6 \lambda r \mu+3 \mu^{2}\right)}{(2 \lambda r-3 \mu)(r+\mu) r^{2}} \chi=0
$$

A straightforward computation shows that this equation has, in general, two independent solutions, one regular and the other with logarithmic divergences for $r=0$, and for $r=-\mu$. The regular solution is a polynomial of order $\ell+2$, given by,

$$
Q(r)=\sum_{i=1}^{\ell+2} q_{i} r^{i}
$$

with

$$
q_{i}=\frac{(i-2) \Gamma(\ell+i-1)[(i-4) \ell(\ell+1)+i-1]}{3 \Gamma(\ell-i+3) \mu^{i-1}[\Gamma(i)]^{2}}
$$

The general solution of (22) may be written in the form,

$$
\chi(r)=C_{1} Q(r)+C_{2} Q(r) \int_{C_{3}}^{r} \frac{y(2 \lambda y-3 \mu)}{(y+\mu) Q(y)^{2}} d y
$$

where $C_{1}, C_{2}$ and $C_{3}$ are constants. Notice that this expression contains effectively only two arbitrary constants, because a change in $C_{3}$ amounts only to a change in $C_{1}$. We also remark that, since all solution of (22) are regular for $r \geq 0$, we must have $Q(r)>0$ for $r>0$, for otherwise a zero in $Q(r)$ would introduce singularities in $\chi$ through the integral term in (25), which must be absent in the general solution of (22).

\section{A. Boundary conditions}

Equations $(24,25)$ provide the general time independent solutions for the perturbative zero mode, but we still need to specify boundary conditions. The question is then if these solutions, perhaps for some particular choices of $C_{1}$ and $C_{2}$, are acceptable as perturbations of the negative mass Schwarzschild metric. As a possible criterion, we explore the change in a particular curvature invariant, which we choose to be the Kretschmann invariant $\mathcal{K}$, given by,

$$
\mathcal{K}=R_{a b c d} R^{a b c d}
$$

where $R_{a b c d}$ is the Riemann curvature tensor. Replacing (24)-(25) in the appropriate expression for $\mathcal{K}$, linearized to first order in the perturbations, we find that, in the $r \simeq 0$ limit

$$
\begin{aligned}
\mathcal{K}=\frac{12 \mu^{2}}{r^{6}} & -\epsilon C_{2}\left[\frac{36 \mu^{2}}{r^{7}}+\mathcal{O}\left(\frac{\ln (r)}{r^{5}}\right)\right] Y_{(\ell, m)} \\
& -\epsilon C_{1}\left[\frac{\ell\left(\ell^{2}-1\right)(\ell+1)(\ell+2)\left(\ell^{2}+\ell+4\right)\left(\ell^{2}+\ell-1\right)}{9 \mu^{2} r^{3}}+\mathcal{O}\left(\frac{1}{r^{2}}\right)\right] Y_{(\ell, m)}
\end{aligned}
$$


We must set $C_{2}=0$ to ensure that the first order term does not grow faster than the zero order term as $r \rightarrow 0^{+}$. On the other hand, if we compute $\mathcal{K}$ for large $r$, we obtain

$$
\begin{aligned}
\mathcal{K}=\frac{12 \mu^{2}}{r^{6}}-\epsilon C_{1}\left[\left(\frac{2 \Gamma(2 \ell+1)}{\mu^{\ell} \Gamma(\ell-1)^{2}}\right) r^{\ell-5}+\mathcal{O}\left(r^{\ell-4}\right)\right] Y_{(\ell, m)} & \\
& +\epsilon C_{2}\left[\frac{K_{\ell}}{r^{\ell+7}}+\mathcal{O}\left(\frac{1}{r^{\ell+8}}\right)\right] Y_{(\ell, m)},
\end{aligned}
$$

where

$$
K_{\ell}=\frac{3 \sqrt{\pi} \Gamma(\ell)\left(3 \ell^{2}+5 \ell+8\right)(\ell+1)(\ell+2) \mu^{\ell+2}}{4^{\ell} \Gamma(\ell+1 / 2)(\ell-1)^{2}},
$$

then $C_{1}$ must also be set to zero in order to keep the perturbation smaller than the zero order term at large $r$. We must, therefore, conclude that there is no (non trivial) choice of $C_{1}$ and $C_{2}$ for which a static perturbation will remain small in the entire range of $r$.

\section{B. A physical interpretation for the zero modes}

Even though there are problems in interpreting the zero modes given by (23)-(25) as perturbations of the negative mass Schwarzschild metric, they are static solutions of the linearized equations and, therefore, their physical interpretation is of interest. One way in which the problem may be analyzed is to consider the Schwarzschild metric in Weyl

coordinates. The general form of the static axially symmetric vacuum metrics in Weyl coordinates is,

$$
d s^{2}=-e^{2 U} d t^{2}+e^{2 k-2 U} d \rho^{2}+\rho^{2} e^{-2 U} d \phi^{2}+e^{2 k-2 U} d z^{2}
$$

where $U=U(\rho, z), k=k(\rho, z)$, and the Einstein equations imply,

$$
\frac{\partial^{2} U}{\partial \rho^{2}}+\frac{1}{r} \frac{\partial U}{\partial \rho}+\frac{\partial^{2} U}{\partial z^{2}}=0
$$

and,

$$
\begin{aligned}
& k,_{\rho}=\rho\left(U,_{\rho}\right)^{2}-\rho(U, z)^{2} \\
& k,_{z}=2 \rho U,_{\rho} U_{, z}
\end{aligned}
$$

where a comma indicates partial derivative. It can be checked that (31) is the integrability condition for the system (32), so that $k$ is obtained by quadratures if $U$, satisfying (31) is given. The latter is the Laplace equation in flat cylindrical coordinates and, as is well known, allows for the construction of static vacuum solutions of the Einstein equations by taking for $U$ different solutions of the Laplace equation corresponding to localized sources. The Schwarzschild space time is recovered by taking for $U$ the Newtonian potential corresponding to uniform line source of finite length, centered at the origin, and laying along the z-axis. The explicit forms for $U$ and $k$ in this case are,

$$
\begin{aligned}
U_{S}(\rho, z) & =\frac{1}{2} \ln \left[\frac{\rho_{+}+\rho_{-}-2 M}{\rho_{+}+\rho_{-}+2 M}\right] \\
k_{S}(\rho, z) & =\frac{1}{2} \ln \left[\frac{\left(\rho_{+}+\rho_{-}\right)^{2}-4 M}{4 \rho_{+} \rho_{-}}\right]
\end{aligned}
$$


where $\rho_{ \pm}=\sqrt{\rho^{2}+(z \pm M)^{2}}$. The constant $M$ is identified with the Schwarzschild mass and can be positive or negative. Suppose now that instead of $U_{S}$ we consider, e.g.,

$$
U(\rho, z)=U_{S}(\rho, z)+U_{1}(\rho, z ; m)
$$

where $U_{1}(\rho, z ; m)$ is a solution of (31) that depends smoothly on a real parameter $m$ such that,

$$
U_{1}(\rho, z ; 0)=0
$$

This implies that $U(\rho, z)$ given (34) satisfies also (31) and can be used to construct static vacuum solutions of the Einstein equations. But, in accordance with (35), these solutions, at least locally, can be made to approach the Schwarzschild metric arbitrarily closely, by taking $m$ small enough. This may fail, and in general it does, when we approach the line source of $U_{S}$. This line source corresponds to the regular horizon for $M>0$ and, therefore, a singularity in $U_{1}$ destroys that regularity, and $U_{1}$ cannot be considered globally as a "small" perturbation. Similarly, for $M<0$, the line source corresponds to a naked singularity, and a singularity in $U_{1}$ may modify its structure, and can make it even "harder". To illustrate this discussion we consider for $U_{1}$ the form,

$$
U_{1}(\rho, z ; m)=\frac{m}{\sqrt{\rho^{2}+z^{2}}}
$$

This represents (in Weyl coordinates) a point source at the origin. The resulting equations for the modified $k(\rho, z)$ may be solved explicitly, for any $m$, but we are only interested in the change to order $m$. Actually what we are interested in is the form of the metric in Schwarzschild, rather than Weyl coordinates. The coordinate change that accomplishes this is well known (see, e.g. [8], for details). Performing it we find that in Schwarzschild coordinates the modified metric, to first order in $m$, takes the form,

$$
\begin{aligned}
g_{t t} & =-1+\frac{2 M}{r}-\frac{2 m(r-2 M)}{r \sqrt{M^{2} \cos ^{2} \theta+r^{2}-2 M r}} \\
g_{r r} & =\frac{r}{r-2 M}+\frac{2 m r\left(2 r-3 M-2 \sqrt{M^{2} \cos ^{2} \theta+r^{2}-2 M r}\right)}{(r-2 M) \sqrt{M^{2} \cos ^{2} \theta+r^{2}-2 M r} M} \\
g_{\theta \theta} & =r^{2}+\frac{2 m r^{2}\left(2 r-3 M-2 \sqrt{M^{2} \cos ^{2} \theta+r^{2}-2 M r}\right)}{M \sqrt{M^{2} \cos ^{2} \theta+r^{2}-2 M r}} \\
g_{\phi \phi} & =r^{2} \sin ^{2} \theta-\frac{2 m r^{2} \sin ^{2} \theta}{\sqrt{M^{2} \cos ^{2} \theta+r^{2}-2 M r}}
\end{aligned}
$$

This metric may be expanded now in the Regge-Wheeler-Zerilli functions. For the metric in the form (37), in the notation of [3], the only non vanishing functions are $H_{0}, H_{2}, K$, and $G$, corresponding to even (scalar) perturbations for even $\ell$, as the result of the symmetry of $U_{1}(\rho, z ; m)$ under $z \leftrightarrow-z$. This is not in the Regge-Wheeler gauge, but, from gauge invariance [10], the function $\psi(r, t)$ is in this case given by,

$$
\begin{aligned}
\psi(r, t) & =\frac{1}{\lambda r+3 M} \chi(r, t) \\
\chi(r, t) & =\frac{2 r(r-2 M)}{\ell(\ell+1)}\left(H_{2}-r \frac{\partial K}{\partial r}-\frac{(r-3 M) K}{r-2 M}\right)+r^{2}\left(K+(r-2 M) \frac{\partial G}{\partial r}\right)
\end{aligned}
$$


Going back to (37), if we specialize to $\ell=2$, we find,

$$
\begin{aligned}
H_{2} & =\frac{\sqrt{5 \pi} m(2 r-3 M)}{2 M^{4}}\left[\left(2 M^{2}-6 M r+3 r^{2}\right) \ln \left(1-\frac{2 M}{r}\right)+6(r-M) M\right] \\
K & =\frac{\sqrt{5 \pi} m}{4 M^{4}}\left[\left(3 r^{3}-15 r^{2} M+16 r M^{2}-2 M^{3}\right) \ln \left(1-\frac{2 M}{r}\right)-24 r M^{2}+10 M^{3}+6 r^{2} M\right] \\
G & =\frac{\sqrt{5 \pi} m}{12 M^{4}}\left[3(M-r)\left(2 M^{2}-2 M r+r^{2}\right) \ln \left(1-\frac{2 M}{r}\right)+2 M\left(6 r M-7 M^{2}-3 r^{2}\right)\right]
\end{aligned}
$$

and the corresponding expression for $\chi(r)$ results,

$$
\chi=\frac{\sqrt{5 \pi} m r}{6 \mu^{3}}\left[3\left(4 r^{3}-6 r^{2} \mu+3 \mu^{3}\right) \ln \left(1+\frac{\mu}{r}\right)-12 \mu r^{2}+24 \mu^{2} r-13 \mu^{3}\right]
$$

which coincides with (23)-(25) for an appropriate choice of integration constants. Similar results hold for the other even values of $\ell$. Non vanishing contributions for odd $\ell$ may be obtained starting from a different choice of $U_{1}(\rho, z ; m)$ that makes it odd under $z \longleftrightarrow-z$, with analogous results regarding the corresponding zero modes. Therefore, the zero modes are the linearized expressions for the modifications that result in the Schwarzschild metric by the addition of different (static) multipoles. We stress again that these modifications approach only locally the Schwarzschild metric, because they modify non trivially the structure of its singularity. We shall add more comments on this feature in Section VIII.

\section{TIME DEPENDENT SOLUTIONS}

Going back to (17), we consider now the general, time dependent case, with $\omega \neq 0$. We are interested in solutions that are regular in $0 \leq r \leq \infty$. Therefore, we need to consider the behavior of $\chi_{1}$ near the singular points $r=0, r=3 \mu /(2 \lambda)$ and $r=\infty$. First we find that near $r=0$, any solution of (17), for any $\omega$, is of the form,

$$
\chi(r) \simeq C_{1} r+C_{2} r \ln (r)+\ldots
$$

where $C_{1}$ and $C_{2}$ are constants, and $\ldots$ indicates terms that vanish faster for $r=0$. This implies that all solutions of (17) vanish for $r=0$, but, in general, $d \chi / d r$ diverges as $\ln (r)$. On account of our previous discussion regarding acceptability of the perturbations, this means that we need to impose the condition that this divergence is absent, and therefore, only the regular solution associated with $C_{1}$ can be considered for the stability analysis. This fixes our boundary condition for $r=0$. The behavior at $r=\infty$ is also simple. For large $r$, the general solution of (17) is of the form (55) [11]. We remind the reader that, in view of Lemma 2 in Section III, $\chi(r)$ is analytic at $r=3 \mu /(2 \lambda)$

From the assumed time dependence, positive values for $\omega^{2}$ correspond to an oscillating, and therefore stable, behavior for the perturbations. The interesting range for instability is, therefore, $\omega^{2}<0$. On account of (13), the solutions for $\omega^{2}<0$ will grow unboundedly for large $r$, unless we can eliminate the exponentially divergent terms. In the following section we analyze this problem in detail. 


\section{UNSTABLE SOLUTIONS}

Unstable modes, correspond to solutions of (17) with $\omega^{2}=-k^{2}$, that satisfy the regularity condition for $r=0$, and are exponentially decreasing for large $r$. Therefore, finding these solutions turns this equation into a boundary value problem, determining the appropriate value or values of $k$, if they exist.

As a first step, we considered a "shooting" approach, and, using a high precision numerical method, we found, for $\ell=2$, a numerical solution for $k \simeq 4 / \mu$. Since this value was so close to an integer times $1 / \mu$, we replaced $k=4 / \mu, \ell=2$ in (17), and found that indeed it has a rather simple solution for this value of $k$. Repeating the procedure for $\ell=3$, we found again a solution for $k=20 / \mu$. This suggested the possibility that we could construct solutions for all $\ell$, by a simple generalization of the results for $\ell=2$, and $\ell=3$. This was effectively the case, and we found that,

$$
\chi(r)=r e^{-k r}(r+\mu)^{k \mu}\left[C_{1}+C_{2} \int_{C_{3}}^{r} \frac{(2 \lambda y-3 \mu)^{2} e^{2 k y}}{(y+\mu)^{2 k \mu+1} y} d y\right]
$$

where $C_{1}, C_{2}$, and $C_{3}$ are arbitrary constants, is a solution of (17) with $\omega^{2}=-k^{2}$, provided the constant $k$ is given by

$$
k=\frac{2 \lambda(\lambda+1)}{3 \mu}=\frac{(l-1) l(l+1)(l+2)}{6 \mu}
$$

and, therefore, $k \mu$ is an integer for all $\ell$. Notice that (42) effectively contains only two arbitrary constants, because any change in $C_{3}$ can be compensated by a corresponding change in $C_{1}$.

It is easy to see that the integral in (42) contributes logarithmic divergences for $r=0$, and an exponentially increasing factor for $r \rightarrow \infty$. Therefore if we choose $C_{2}=0$, we find that

$$
\zeta(t, r)=C_{1} r e^{-k r}(r+\mu)^{k \mu} e^{k t}
$$

with $k$ given by (43), is a solution of (17), representing the general expression for the regular, bounded, unstable modes. Moreover, this result implies that all scalar modes (i.e. for all $\lambda$ ) of the Schwarzschild negative mass naked singularity are linearly unstable.

As a check of the consistency of our results, we replace (44) in (12), and this in(3), and obtain

$$
\begin{aligned}
K(t, r) & =-\frac{(\lambda+1)(r+\mu)^{k \mu}}{3 \mu} \exp [k(t-r)] \\
H_{1}(r, t) & =-H_{2}(t, r)=-\frac{\lambda(\lambda+1)[2(\lambda+1) r+3 \mu] r(r+\mu)^{k \mu-1}}{9 \mu^{2}} \exp [k(t-r)]
\end{aligned}
$$

which shows explicitly the regularity of the solution of the perturbative equations in the range $0 \leq r<+\infty$. The perturbations of the curvature are also well behaved. Replacing in the Kretschmann scalar, we find that it is unchanged from the unperturbed value, to order $\epsilon$. Furthermore, a complete set of quadratic algebraic invariants of the Riemann tensor $[6,7]$ was computed to find that they are all invariant to first order in $\epsilon$. However, we have found non trivial first order contributions to the differential invariants

$$
\begin{aligned}
R_{a b c d ; e} R^{a b c d ; e}= & \frac{180 \mu^{2}(r+\mu)}{r^{9}}-\epsilon \frac{5 \ell(\ell-1)(\ell+1)(\ell+2)}{r^{8}} Y_{\ell m}(\theta, \phi) \\
& \times[r \ell(\ell+1)+3 \mu](r+\mu)^{k \mu} \exp [k(t-r)]
\end{aligned}
$$


and

$$
\begin{aligned}
& R_{a b c d ; e f} R^{a b c d ; e f}=\frac{90 \mu^{2}\left(65 \mu^{2}+120 \mu r+56 r^{2}\right)}{r^{12}} \\
& -30 \epsilon k \frac{(r+\mu)^{k \mu} \exp [k(t-r)]}{r^{11}} Q_{\ell}(r) Y_{\ell m}(\theta, \phi) \\
& \begin{array}{r}
Q_{\ell}(r)=\left(l^{2}(l-1)(l+2)(l+1)^{2} r^{3}+3 \mu l(l+1)\left(l^{2}+l+14\right) r^{2}\right. \\
\left.+3 \mu^{2}\left(17 l^{2}+17 l+56\right) r+180 \mu^{3}\right)
\end{array}
\end{aligned}
$$

and we may expect non trivial contributions in some of the many other differential invariants. Note that, both for (46) and (47), the $\mathcal{O}(\epsilon)$ term grows slower than the $\mathcal{O}\left(\epsilon^{0}\right)$ term as $r \rightarrow 0^{+}$ limit, and also as $r \rightarrow \infty$.

\section{COMPARISON WITH PREVIOUS RESULTS}

In [2], the Hilbert space

$$
\mathcal{H}=L^{2}\left(r^{*}, d r^{*}\right)
$$

is introduced together with Zerilli's equation (11). Since the LHS of (11) is a self adjoint operator in $\mathcal{H}$, it admits a basis of eigenfunctions that allow to evolve any initial data $\left.\left(\psi, \partial_{t} \psi\right)\right|_{t=0} \in \mathcal{H} \otimes \mathcal{H}$. It was also shown in [2] that (for $\left.\ell=2\right)$ there is a $\omega^{2}=0$ solution $\hat{\psi}_{o} \in \mathcal{H}$ of (11). It is important to review at this point how $\hat{\psi}_{o}$ is constructed. For negative $M=-\mu / 2$ the $\ell=2$ Schrödinger potential

$$
V=2\left(1+\frac{\mu}{r}\right) \frac{4 r^{2}(3 r-3 \mu / 2)+\frac{9}{4} \mu^{2}(2 r-\mu / 2)}{r^{3}\left(2 r-\frac{3 \mu}{2}\right)^{2}},
$$

is singular at $r_{c} \equiv 3 \mu / 4$. The general solution of (11) for $\omega=0[2]$

$$
\begin{aligned}
\psi_{o}=C_{3} \frac{r\left(3 \mu^{3}-6 r^{2} \mu+4 r^{3}\right)}{\mu^{3}(4 r-3 \mu)} & \\
& \quad+C_{4}\left[\frac{r\left(13 \mu^{3}+12 r^{2} \mu-24 \mu^{2} r\right)}{3 \mu^{3}(4 r-3 \mu)}-\frac{r\left(3 \mu^{3}-6 r^{2} \mu+4 r^{3}\right)}{\mu^{3}(4 r-3 \mu)} \log \left(\frac{r+\mu}{r}\right)\right]
\end{aligned}
$$

lies outside $\mathcal{H}$, however, for $C_{3} / C_{4}=\log (7 / 3)-4 / 9 \equiv q_{o}$, both $\psi_{o}$ and its first derivative vanish at $r_{c}[2]$, then $\psi_{o}$ and can be matched at $r_{c}$ to the trivial $\omega=0$ solution giving

$$
\hat{\psi}_{o}(r)= \begin{cases}\psi_{o}(r) & , 0 \leq r<r_{c} \\ 0 & , r>r_{c}\end{cases}
$$

Note that $\hat{\psi}_{o}$ is an $\omega=0$ solution in $\mathcal{H}$, whose second derivative has a discontinuity at $r=r_{c}$. The existence of such a solution signals a critical value of $q \equiv C_{3} / C_{4}$ separating unstable $\left(\omega^{2}<0\right)$ from stable solutions for the Zerilli equation in $\mathcal{H}$.

In the previous section we exhibited a $C^{\infty}$ perturbed metric which is an unstable solution of the linearized Einstein's vacuum equation around a negative mass Schwarzschild spacetime, 
and showed that it satisfies appropriate boundary conditions, both at the singularity and at infinity. However, we have not given a procedure to evolve arbitrary perturbations. The reason why we have not adopted the Hilbert space (49) is that, although (49) is a natural choice in other contexts, generic linear perturbations (2) of the metric of a negative mass Schwarzschild spacetime lye outside $\mathcal{H}$, since the integral

$$
\begin{aligned}
\left(\psi_{Z}, \psi_{Z}\right)=\int_{0}^{\infty}\left|\psi_{Z}\right|^{2} \frac{d r}{(1+\mu / r)} & \\
= & \int_{0}^{\infty}\left|\frac{r(r+\mu)}{6(r-3 \mu / 4)}\left(H_{2}-r \frac{\partial K}{\partial r}\right)+\frac{r}{3} K\right|^{2} \frac{d r}{(1+\mu / r)}
\end{aligned}
$$

diverges due to the pole at $r=r_{c}=3 \mu / 4$ (here we have specialized to $\ell=2$ and used (9) to write the $L^{2}\left(r^{*}, d r^{*}\right)$ norm of the perturbation in terms of the perturbed metric elements, which may be arbitrary $C^{2}$ functions for $\left.r \in(0, \infty)\right)$. Even compactly supported metric perturbations are excluded as initial data in the formalism developed in [2], unless they meet the rather artificial requirement that, for every $\ell \geq 2$, its $\ell-t h$ harmonic projection satisfies

$$
\left.\left(H_{2}^{\ell}-r \frac{\partial K^{\ell}}{\partial r}\right)\right|_{\left(r=\frac{3 \mu}{2(\ell-1)(\ell+2)}\right)}=0
$$

The Zerilli function $\psi_{Z}$ is extremely useful because it reduces a complicated PDE system to a much simpler differential equation. However, care must be exercised when deciding what kind of solutions of this differential equation one is interested in, since $\psi_{Z}$ has, for the negative mass Schwarzschild spacetime, a built in singularity, as seen, e.g., from (9). As an example, it follows from (2) and (3), that the perturbed metric from (52) has a discontinuity at $r=r_{c}$. In particular, it will not give a linearized solution of the vacuum Einstein's equations, in spite of being a solution of Zerilli's equation. This implies that, although (52) gives a marginally stable solution for the Zerilli problem in $\mathcal{H}=L^{2}\left(r^{*}, d r^{*}\right)$, it does not prove the existence of a marginally stable solution of the linearized Einstein's equations we are dealing with, since only $C^{4}$ solutions to the Zerilli equation are relevant to the linearized gravity problem. Our choice of initial data set is that $\left.\zeta\right|_{t=0}$ and $\partial \zeta /\left.\partial t\right|_{t=0}$ be $C^{\infty}$ functions subject to (16). As follows from Lemma 1 in Section III, this is equivalent to requiring that the metric perturbation be $C^{\infty}$. Equation (13) is a hyperbolic differential equation in $(t, r)$ from which the evolution of given initial data for $H_{1}, H_{2}$ and $K$ can be computed. The singularities in the coefficients of $\partial \chi / \partial r$ and $\chi$, for $2 \lambda r=3 \mu$ were shown to be irrelevant in Lemma 2 of Section III. The hyperbolicity of (13), (or the regularity and smoothness of the light cones for the background metric), imply that smooth initial data of compact support given at, say, time $t=t_{0}$, should evolve into initial data also of compact support for $t=t_{1}$, at least for sufficiently small $t_{1}-t_{0}$, then compactly supported smooth $\left.\zeta\right|_{t=t_{o}}$ and $\partial \zeta /\left.\partial t\right|_{t=t_{o}}$ satisfying (16) evolves into a compactly supported $\left.\zeta\right|_{t=t_{1}}$

The foregoing discussion shows that (45) can actually be excited without a fine tuning of the initial condition. We look at solutions of (13) corresponding to a time dependence of the form $\exp (i \omega t)$, with $\omega$ arbitrary complex, without the logarithm singularity $\left(C_{2}=0\right.$ in (41)). A higher order expansion around $r=0$

$$
\tilde{\chi}(r, \omega) \propto\left[r-\frac{\lambda(1+\lambda)}{3 \mu^{2}} r^{3}+\frac{2 \lambda(1+\lambda)}{9 \mu^{3}} r^{4}+\mathcal{O}\left(r^{5}\right)\right]
$$

reveals that there is no term in $r^{2}$, and only the coefficients of $r^{5}$ and higher order depend on $\lambda$ and on (even) powers of $\omega$. We have already shown in Section III (Lemma 2) that 
all solutions of (13) for this type of time dependence are smooth at $r=3 \mu /(2 \lambda)$. We may similarly obtain an asymptotic expansion for these solutions for $r \rightarrow \infty$. These can be written in the form,

$$
\tilde{\chi}(r, \omega) \propto e^{[i k(r-\mu \ln (r+\mu)]}\left[r-\frac{2 i \lambda(1+\lambda)-3 k \mu}{2 k \lambda}+\frac{2 \lambda(1+\lambda)+3 i k \mu}{4 \omega^{2} r}+\mathcal{O}\left(1 / r^{2}\right)\right]
$$

where $k= \pm \omega$, and the remaining coefficients depend only on even powers of $\omega$. For real $\omega$, and large $r$, these solutions represent (modulated) incoming and outgoing waves, depending on the sign of $k / \omega$. Since (13) is linear, any linear superposition of solutions is also a solution. In particular, we may consider superpositions of the form,

$$
\chi(r, t)=\int_{-\infty}^{+\infty} \mathcal{K}(\omega) e^{i \omega t} \tilde{\chi}(r, \omega) d \omega
$$

which provide everywhere regular solutions (including $2 \lambda r=3 \mu$, for a wide variety of functions $\mathcal{K}(\omega)$. An interesting choice is,

$$
\mathcal{K}(\omega)=C e^{-\left(\omega-\omega_{0}\right)^{2} q^{2} / 4}
$$

where $C, \omega_{0}$, and $q$ are constants. Then, for large $q$, we may use a steepest descent estimation of (56) to find that for $\mathcal{K}(\omega)$ of the form (57), near $r=0$ we have,

$$
\chi(r, t) \simeq K_{1} e^{-t^{2} / q^{2}} e^{i \omega_{0} t}\left[r-\frac{\lambda(1+\lambda)}{3 \mu^{2}} r^{3}+\frac{2 \lambda(1+\lambda)}{9 \mu^{3}} r^{4}+\mathcal{O}\left(r^{5}\right)\right]
$$

where $K_{1}$ is a constant, and terms of order $r^{5}$ or higher are polynomial in $t$. This result implies that the amplitude near $r=0$ is strongly suppressed for large $t$. For large $r$, on the other hand, we find,

$$
\begin{aligned}
\chi(r, t) \simeq & K_{2} \exp \left[i \omega_{0} u_{ \pm}\right] \exp \left[-\left(u_{ \pm}\right)^{2} / q^{2}\right] \\
& \times\left[r-\frac{2 i \lambda(1+\lambda)-3 k \mu}{2 k \lambda}+\frac{2 \lambda(1+\lambda)+3 i k \mu}{4 \omega^{2} r}+\mathcal{O}\left(1 / r^{2}\right)\right]
\end{aligned}
$$

where $K_{2}$ is constant, and,

$$
u_{ \pm}(r, t)=t \pm[r-\mu \ln (r+\mu)]
$$

Since this expansion is valid only for large $r$, we find that solutions corresponding to $u_{+}$are strongly suppressed for all $t$, while those for $u_{-}$represent an outgoing wave packet, roughly centered at $t=[r-\mu \ln (r+\mu)$, moving along a null geodesic of the unperturbed spacetime. All these solutions represent the evolution of some smooth initial data that can be obtained from (56), for $t=0$. Clearly, these superpositions display a "stable" behaviour, since they do not contain modes that grow exponentially in time. We notice, however, that we may combine any of these solutions with an arbitrarily small, but nonzero contribution from the unstable mode given by eqs. (44)-(45), to obtain an equally smooth initial data at $t=0$, whose evolution displays the exponentially growing mode. We thus find a very large set of smooth initial data leading to an equally smooth "unstable" behaviour, showing that such data need not be particularly "fine tuned". 
Another issue discussed in [2] is that of the gravitational energy of the perturbation. Here again we find a number of subtleties, apparently peculiar to the negative mass case. The unstable solution (45) lies outside $\mathcal{H}$ in [2], and therefore outside the scope of ref [2]. To no surprise, its "Zerilli energy"

$$
E_{Z}:=\int\left(\left|\frac{\partial \psi_{Z}}{\partial t}\right|^{2}+\left|\frac{\partial \psi_{Z}}{\partial r^{*}}\right|^{2}+V\left|\psi_{Z}\right|^{2}\right) d r^{*}
$$

diverges. It is argued in [2] that $E_{Z}$ agrees with the notion of gravitational energy of a perturbation from second order perturbation theory, i.e.,

$$
E_{G}=-\frac{1}{8 \pi} \int G_{a b}^{(2)} \eta^{a} \zeta^{b} d \Sigma_{(3)}, \quad \eta=f^{-1 / 2} \partial / \partial t, \quad \zeta=\partial / \partial t,
$$

$G_{a b}^{(2)}$ being the second order correction to the Einstein tensor. However, from (45) we get, for the $(\ell, m=0)$ mode,

$$
G_{t t}^{(2) \ell}=\exp (k t)\left[A(r, \ell)\left(Y_{(\ell, 0)}\right)^{2}+B(r, \ell)\left(\frac{\partial Y_{(\ell, 0)}}{\partial \theta}\right)^{2}\right]
$$

where $A$ and $B$ are $C^{\infty}$ functions of $r$ in $(0, \infty)$, falling off exponentially as $r \rightarrow \infty$, and behaving as $c / r^{3}$ for $r \simeq 0$. This implies that both

$$
\int_{0}^{\infty} \frac{r^{2} A}{f} d r \quad \text { and } \quad \int_{0}^{\infty} \frac{r^{2} B}{f} d r
$$

converge, and thus $E_{G}$ is finite for the perturbation (45), showing that $E_{Z} \neq E_{G}$ in the negative mass case.

We may try to find an alternative Hilbert space and energy concept associated to a Zerilli-like equation

$$
\frac{\partial^{2} \phi}{\partial t^{2}}+\mathcal{O}(\phi)=0, \quad \mathcal{O}=A(r) \frac{\partial^{2}}{\partial r^{2}}+B(r) \frac{\partial}{\partial r}+C(r) .
$$

Yet, is not hard to see that, for unstable spacetimes, all unstable modes will either be outside the Hilbert space, or have zero energy. To see this suppose we choose any hermitian product $<,>$ in an appropriate function space under which $\mathcal{O}$ is self-adjoint: $<\phi_{1}, \mathcal{O} \phi_{2}>=<$ $\mathcal{O} \phi_{1}, \phi_{2}>$. Note that the hermitian product may well involve $\phi$ and $r$-derivatives of $\phi$. Adding $0=\left\langle\phi_{t}, \phi_{t t}+\mathcal{O} \phi>\right.$ to its complex conjugated, and using the self adjointness of $\mathcal{O}$, we arrive to a conserved "energy"

$$
\frac{d E}{d t} \equiv \frac{d}{d t}\left[<\phi_{t}, \phi_{t}>+<\phi, \mathcal{O} \phi>\right]=0
$$

This is essentially a generalization of the argument used in [5] to obtain the conserved energy (61) above. We would like to use it now to prove that, if the underlying spacetime is unstable, the unstable modes will either be non normalizable (and then $E$ in (65) undefined, as happens with the energy $E_{Z}$ for (45)), or will trivially vanish. In fact, suppose there is a solution $\phi(t, r)=\exp (\kappa t) \phi_{o}(r), \kappa>0$ and assume $\phi_{o}$ normalizable under $<,>$, so that the energy in (65) is well defined. Since

$$
E(t)=e^{2 \kappa t}\left[\kappa^{2}<\phi_{0}, \phi_{0}>+<\phi_{0}, \mathcal{O} \phi_{0}>\right]
$$

does not depend on $t$, it has to vanish. 


\section{COMMENTS AND CONCLUSIONS}

In this article we have shown that even if we only allow for the most restrictive boundary conditions, both as $r \rightarrow 0^{+}$and $r \rightarrow \infty$, the perturbation equations for the negative mass Schwarzschild metric contain, for every angular mode, solutions that grow exponentially in time. These solutions represent everywhere regular perturbations that can be made initially globally arbitrarily small and, therefore, imply that the metric is unstable at the perturbative level, for all angular modes. An interesting technical point of our derivations was the introduction of a modification of the standard Regge-Wheeler-Zerilli method, that led to equation (17) and its rather unusual properties. Our analysis of the zero modes indicates that rather than perturbations they should be considered as linearized approximations for exact static metrics that locally, but not globally approach the negative mass Schwarzschild metric. Although asymptotically flat, they actually contain a naked singularity where the curvature diverges even faster than for the Schwarzschild metric. From the perspective of the present analysis, these spacetimes should also be considered unstable, since, if they are considered as part of the unperturbed metric, one would be allowed to relax the boundary conditions at $r=0$, and as can be seen from the derivations in Section VI, this would lead to unstable solutions for any $k=\sqrt{-\omega^{2}}$. Our results also suggest that, even though the vector (linearized) perturbations have been shown to be stable [2], one would expect that these would also become unstable through the coupling to the scalar modes. Since vector modes are related to rotation, this again suggests that rotation would not make the singularity more stable, although going through this in detail would require higher order perturbation theory, or, perhaps, some application of the Teukolsky equation.

Finally, there is an obvious implication of our results to the cosmic censorship conjecture, since the linear instability would imply that the negative mass Schwarzschild cannot be the final stage of an evolving spacetime.

\section{Acknowledgments}

We acknowledge fruitful correspondence with and comments from G. Gibbons, A. Ishibashi and S. Hartnoll that helped us clarify the main differences between their approach in [2] and ours. G.D. thanks the KITP at UCSB, where part of this work was done, for hospitality. This work was supported in part by grants of CONICET (Argentina) and the Universidad Nacional de Córdoba. It was also supported in part by grant NSF-INT-0204937 of the National Science Foundation of the US. The authors are supported by CONICET (Argentina).

[1] Carroll S M (2003) Phys. Rev. C68 023509, astro-ph/0301273.

Gibbons G W (2003), hep-th/0302199.

Caldwell R R (2002) Phys. Lett. B545 23, astro-ph/9908168.

[2] Gibbons G W, Hartnoll D and Ishibashi A 2005 Prog.Theor.Phys. 113 963-978, hepth/0409307.

[3] Regge T and Wheeler J A 1957 Phys. Rev. 108, 1063.

[4] Zerilli F J 1970 Phys. Rev. Lett 24737. 
[5] Chandasekhar S, The mathematical theory of black holes, Oxford University Press, 1992.

[6] Carminati J and McLenaghan R G 1991 Jour.Math.Phys 33, 3135.

[7] Zakhary E and McIntosh C B G 1997 Gen.Rel.Grav 29, 539.

[8] Stephani H, Kramer D, MacCallum M, Hoenselaers C and Herlt E Exact Solutions to Einstein's field equations 2nd ed (2003).

[9] Braun M Differential equations and their applications (fourth edition) Springer-Verlag 1993.

[10] Moncrief V (1974) Ann. Phys3 88323.

[11] Notice that the leading term in $r$ in (55) can be traced to the transformation (12), since, for any sign of $M$, the Zerilli equation for $\psi(t, r)$ approaches the one dimensional wave equation, and $\psi(t, r) \rightarrow \exp (i \omega t)$. 